\title{
L'ente quale centro di imputazione di responsabilità nel processo penale ai sensi del d.lgs. 231/2001
}

\author{
Chiara Tebano $0^{\circ}$
}

\section{Riassunto}

L'autore esamina il contenuto del decreto legislativo 8 giugno 2001, n. 231, in materia di responsabilità amministrativa delle persone giuridiche, delle società e delle associazioni anche prive di personalità giuridica, sotto il profilo della natura della responsabilità normativamente prevista a carico dell'ente.

Giurisprudenza e dottrina si sono a lungo interrogate, con diversi approdi interpretativi, in ordine alla natura e al fondamento della responsabilità dell'ente (se penale, amministrativa o appartenente ad un tertium genus), anche in considerazione delle ricadute che ne derivano sul piano pratico.

\section{Résumé}

L'auteur examine le décret législatif $\mathrm{n}^{\circ} 231$ du 18 Juin 2001, concernant la responsabilité administrative des personnes juridiques, des entreprises et des associations éventuellement dépourvues de la personnalité juridique conformément à la réglementation.

La jurisprudence et la doctrine se sont toujours chargées d'explorer, en utilisant des approches différentes, le caractère de la responsabilité de l'entreprise (pénale, administrative ou appartenant à une troisième catégorie), compte tenu également des éventuels effets négatifs.

\section{Abstract}

The author examines the contents of the legislative decree 8 June 2001 n. 231 concerning the administrative responsibility of legal persons, companies and associations also without legal personality according to the established regulations.

Case-law and the legal doctrine have always been investigating, through different interpretative approaches, the nature regarding the legal person responsibility (criminal, administrative or belonging to a tertium genus) taking also into account the potential negative effects in practice.

Key words: administrative responsibility; legal persons; Italian legislative decree n. 231/2001; nature of responsibility.

\section{L'ente quale nuovo soggetto del procedimento e del processo penale.}

Il vigente codice di procedura penale dedica, in apertura, il libro I ai soggetti del procedimento e del processo penale, trattando distintamente del Giudice, del Pubblico Ministero, della Polizia Giudiziaria, dell' imputato, della parte civile, responsabile civile e civilmente imputato per la pena pecuniaria, della persona offesa dal reato, ed infine del difensore.

Pare tuttavia potersi attualmente riconoscere la presenza nel processo penale di un ulteriore soggetto rispetto a quelli ora citati e specificamente disciplinati dal codice, e precisamente l'ente - o meglio, secondo la rubrica del d.lgs. 231/2001, le "persone giuridiche, società e associazioni anche prive di personalità giuridica" -, le quali (con la espressa esclusione dello Stato, degli enti pubblici territoriali, degli

\footnotetext{
- E' iscritta all'Ordine degli Avvocati di Bologna dal 1995 e all'Albo Speciale degli Avvocati Cassazionisti dal 2010. Perfezionata presso l'Universita' Statale di Milano in "Questioni controverse in materia di criminalita' dei colletti bianchi" e "Il contenzioso avanti le Corti Europee: la Corte Europea dei diritti dell'Uomo". Master in "Progettazione del Modello Organizzativo ex d.lgs. 231/2001".
} 
altri enti pubblici non economici e degli enti che svolgono funzioni di rilievo costituzionale), a far data dal 4 luglio 2001, epoca dell'entrata in vigore del decreto legislativo 231, sono soggetti passivi di una responsabilità che la norma espressamente definisce "amministrativa", di cui si cercheranno, senza naturalmente alcuna pretesa di esaustività, di evidenziare la effettiva natura e i relativi aspetti problematici. Vanno innanzitutto chiarite le ragioni della rilevanza penalistica e processualpenalistica di tale forma di responsabilità, che risiedono nella circostanza per cui essa presuppone ed è conseguente alla commissione di un reato - si vedrà poi di quali tipologie di reato si tratta - da parte di soggetti ( persone fisiche ) legati all'ente da rapporti meglio definiti nel decreto medesimo, e precisamente all' art. 5 dello stesso.

Tale responsabilità, inoltre, come espressamente previsto dagli artt. 34, 35 e 36 del decreto, va accertata all' interno del processo penale : la competenza a conoscere e decidere in ordine agli illeciti amministrativi che si assumono commessi dall' ente è infatti attribuita al giudice penale competente in relazione al reato dal quale essi dipendono; accanto alle disposizioni di procedura espressamente dettate dal decreto è prevista l'applicabilità - "in quanto compatibili" - delle disposizioni del codice di procedura penale e delle relative norme di attuazione, di coordinamento e transitorie; è infine stabilito che - salva sempre la compatibilità delle medesime - all'ente si applichino le disposizioni processuali relative all' imputato.

E' chiaro, quindi, sin da queste primissime indicazioni in ordine alla forma di responsabilità così introdotta e alle modalità del suo accertamento, come principi ed istituti di natura civilistica, amministrativa e penale nonchè processuale penale siano destinati ad interagire tra loro, determinando questioni di non sempre agevole soluzione interpretativa.

\section{L'introduzione nell'ordinamento italiano della responsabilità amministrativa delle persone giuridiche: origine e ragioni di politica legislativa.}

L'introduzione nel nostro ordinamento, con il decreto 231/2001, del principio della responsabilità da reato degli enti giuridici, è stata da molti vista come una sorta di rivoluzione copernicana rispetto al brocardo per cui 'societas delinquere non potest' e, di conseguenza, 'puniri non potest, risalente alla tradizione romanistica nella quale si era affermata la concezione, poi trasferita nel diritto italiano, nel quale è stato poi costituzionalizzata, della natura individuale e personale della responsabilità penale, che ha sempre, sinora, escluso la configurabilità di responsabilità penale in capo a soggetti diversi dalle persone fisiche.

Tale principio, per varie ragioni, ha ottenuto riconoscimenti anche nel periodo illuministico, senza tuttavia che questo impedisse poi che, tra la fine dell' 800 e l' inizio del ' 900 , nei paesi di common law - in particolare, negli Stati Uniti prima e quindi nel mondo anglosassone in genere - esso venisse progressivamente abbandonato per far spazio all'opposto principio della responsabilità criminale delle persone giuridiche.

Si ritiene che tale cambiamento di prospettiva, avvenuto nei paesi di common law con notevole anticipo rispetto a quelli continentali, sia dovuto essenzialmente a ragioni di carattere storico/economico e, quindi, culturale, legate alla maggiore e più risalente crescita industriale avvenuta in quei Paesi, e quindi al più ampio diffondersi del fenomeno societario, favorite poi dalla circostanza per cui la matrice giurisprudenziale (anche se completata poi da interventi di diritto positivo) del diritto proprio di quegli 
ordinamenti abbia consentito un più rapido adeguamento della disciplina normativa alla realtà economica e sociale.

Nell'ordinamento italiano invece l'introduzione di tale forma di responsabilità è avvenuta solo con il decreto in commento, adottato - peraltro con significativo ritardo - in osservanza di obblighi internazionali assunti dal nostro Paese, in particolare con la Convenzione OCSE sottoscritta a Parigi il 17 dicembre 1997.

La legge delega n. $300 / 2000$, in forza della quale il decreto 231 è stato emanato, è appunto legge di ratifica ed esecuzione nel nostro ordinamento della citata Convenzione.

Quest'ultima, avente ad oggetto la lotta alla corruzione dei pubblici ufficiali stranieri nelle operazioni economiche internazionali, fa obbligo, tra l' altro, agli Stati aderenti, di perseguire non solo la persona fisica responsabile della corruzione, ma anche, in maniera diretta, le imprese coinvolte, in ragione degli scopi di regolazione della condotta degli affari e di disciplina della concorrenza che la Convenzione si prefigge.

Peraltro, se da un lato la Convenzione ha impegnato i Paesi aderenti a perseguire, in forme che siano efficaci e dissuasive, anche le persone giuridiche, dall'altro tuttavia non ha imposto ai legislatori nazionali scelte predeterminate quanto al tipo di responsabilità da collegare ai fatti - o meglio ai misfatti - dell'ente.

I codici penale e di procedura penale italiani sono, come noto, strutturati in funzione della responsabilità della sola persona fisica.

Si è posto, pertanto, per il nostro legislatore il problema di come disciplinare, in ossequio agli impegni internazionali, la responsabilità di quelle altre entità, non umane e non individuali, che tuttavia sono presenti nella realtà sociale, economica e politica e sono anzi in grado di condizionarla in maniera significativa.

La scelta compiuta con il decreto 231/2001, come detto, è stata nel senso di una responsabilità dell'ente espressamente qualificata come amministrativa, ma dipendente dalla commissione di specifiche ipotesi di reato contemplate nel decreto medesimo - secondo il cd. 'modello chiuso' - e da accertarsi in sede penale (sede correttamente considerata, anche in ambito internazionale, come assistita dalle maggiori garanzie), in concomitanza (con il cd. simultaneus processus, secondo quanto previsto dall'art. 38 del decreto) con il procedimento penale instaurato nei confronti dell' autore del reato da cui dipende l'illecito dell'ente e da parte del medesimo Giudice competente a conoscere di tale ultimo reato.

Sotto il profilo sanzionatorio, poi, sono previste a carico dell'ente misure di carattere sia patrimoniale che interdittivo (anche di natura cautelare) estremamente incisive e tali da poter condizionare in maniera determinante sia il patrimonio che la capacità di azione dello stesso; sanzioni quindi che, se pure espressamente definite, sul piano normativo, come amministrative, potrebbero tuttavia essere considerate, dal punto di vista sostanziale e sulla base della giurisprudenza della Corte Europea dei Diritti dell’Uomo in relazione alla cosiddetta "materia penale", di carattere effettivamente penale.

\section{La questione della natura della responsabilità dell'ente.}


Sin dal momento dell' entrata in vigore del decreto si è pertanto assistito al contrapporsi di diverse teorie in ordine alla natura della responsabilità dell' ente così introdotta.

Senza nulla voler togliere all' interesse rivestito delle questioni dogmatiche, la questione rileva, per chi si trovi a dover fare applicazione pratica delle norme, essenzialmente per determinare se a tale tipo di responsabilità debba applicarsi (qualora si ritenga vertere in materia di responsabilità penale) il principio di colpevolezza, nonché istituti previsti dal diritto penale sostanziale, quali, ad esempio, la sospensione condizionale della pena o la disciplina in materia di aggravanti e attenuanti, nonché infine - per citare alcune tra le questioni che hanno occupato la giurisprudenza e gli interpreti - per stabilire se sia ammissibile o meno la costituzione di parte civile nei confronti dell'ente, e se vi sia o meno un rapporto di solidarietà tra l'ente e l'imputato persona fisica sul punto della confisca .

Tre sono i punti di vista da varie parti sostenuti nel corso del tempo, ovvero che si trattasse di una responsabilità sostanzialmente penale (secondo i criteri elaborati dalla Corte Europea dei Diritti dell'Uomo), oppure di una responsabilità amministrativa, così come definita dalla lettera della legge, oppure infine di un cd. tertium genus, che darebbe luogo ad una responsabilità propria, colpevole, per immedesimazione.

Il punto d'arrivo giurisprudenziale sembrerebbe allo stato attuale essere rappresentato dalla pronuncia delle SS.UU. penali Gubert (sentenza n. 10561/2014, pronunciata il 30/1/2014 e depositata il 5/3/2014), la quale testualmente afferma sul punto: "Nel vigente ordinamento, è prevista solo una responsabilità amministrativa e non una responsabilità penale degli enti (ai sensi del d. lgs. 8 giugno 2001, n. 231), sicché comunque la società non è mai autore del reato e concorrente nello stesso".

\section{I criteri oggettivi e soggettivi di ascrizione della responsabilità.}

Veniamo allora ad esaminare i criteri generali ascrittivi della responsabilità dell'ente, così come delineati dal decreto 231 (in particolare, dagli artt. 5, 6 e 7).

L' illecito dell'ente configura una fattispecie complessa che richiede, sul piano oggettivo, innanzitutto la commissione di un reato, che sia integrato nei suoi estremi sia soggettivi che fattuali, e che faccia parte del catalogo, tassativo ma in continua espansione, attualmente contenuto negli articoli da 24 al 25 duodecies del decreto stesso.

Occorre poi che l'autore del reato sia persona (fisica, si intende ) legata all'ente da un rapporto funzionale che può atteggiarsi in due diverse modalità a seconda che ricorra l'ipotesi di cui alla lettera a) ovvero alla lettera b) dell'art. 5 d. lgs. 231/2001; il verificarsi dell'una piuttosto che dell'altra ipotesi comporta altresì differenti conseguenze ed oneri a carico delle parti sul piano probatorio .

Nel primo caso si tratta di quei soggetti che rivestono funzioni di rappresentanza, amministrazione o di direzione dell'ente o di una sua unità organizzativa dotata di autonomia finanziaria e funzionale, ovvero, espressamente, che esercitano, anche di fatto, la gestione e il controllo dell'ente medesimo (i cd. apicali).

Nel secondo si tratta delle persone sottoposte alla direzione e vigilanza dei soggetti sopra indicati (i cd. sottoposti). 
Occorre infine, sempre secondo la previsione del comma 1 dell'art. 5 del decreto, che il reato sia stato commesso nell' interesse o vantaggio dell'ente medesimo - espressamente escludendosi la responsabilità dell'ente, al comma 2 del medesimo articolo, qualora i soggetti sopra indicati abbiano agito nell' interesse esclusivo proprio o di terzi.

Dal punto di vista soggettivo la responsabilità dell'ente si configura quale colpa in organizzazione, ovvero per non essersi adeguatamente organizzato per minimizzare il rischio della commissione dei reati specificamente indicati nel decreto medesimo.

Sul punto appare opportuno svolgere alcune brevi considerazioni.

Come detto, a differenza di altri ordinamenti ( quale ad esempio quello francese ), il nostro legislatore ha optato per un sistema chiuso e quindi per una elencazione tassativa, che si rinviene nel decreto medesimo, dei reati che, soli, possono costituire presupposto di responsabilità amministrativa dell'ente.

Se alcune categorie di reati - ad esempio quelli previsti sin dalla prima versione del decreto, quali i reati contro la pubblica amministrazione e altri successivamente introdotti, quali i reati di criminalità organizzata, reati informatici, reati di falso, reati di terrorismo, reati societari, reati di ricettazione, riciclaggio e reimpiego illecito, ovvero reati di stampo economico - integrando, per la massima parte, figure delittuose dolose non pongono forse significativi problemi di compatibilità con la particolare forma di responsabilità prevista dal decreto 231, è certo invece che numerosi profili problematici si sono manifestati in relazione ad ipotesi colpose di successiva introduzione, e particolarmente quelle relative ai fatti di omicidio colposo e lesioni personali colpose con violazione delle norme in materia di sicurezza e salute sul lavoro (i cd. reati colposi d'evento, di cui all'art. 25 septies del decreto), nonché per alcune ipotesi di reati ambientali .

Si è infatti, sotto questi aspetti, immediatamente verificata la difficoltà di conciliare ipotesi colpose con i criteri dell' interesse o vantaggio per l'ente.

\section{Una breve parentesi in ordine ai reati presupposto di cui all'art. 25 septies d.lgs. 231/2001.}

Sulla premessa, ormai acquisita in giurisprudenza, secondo cui i due criteri, anche in considerazione della lettera della legge, sono da considerarsi alternativi tra loro, si è detto che il criterio del vantaggio ha natura oggettiva e va valutato ex post, mentre quello dell'interesse avrebbe natura soggettiva e sarebbe da valutarsi ex ante, risolvendosi in una "tensione finalistica della condotta illecita dell'autore volta a beneficiare l'ente stesso, in forza di un giudizio ex ante, ossia da riportare al momento della violazione della norma cautelare" (così il Tribunale di Torino, sez. I, sent.10 gennaio 2013, imp. MW Italia s.p.a.).

Ebbene, in materia di delitti colposi d'evento commessi con violazione delle norme in materia di sicurezza e salute sul lavoro, si è detto che l'unico criterio applicabile nei confronti dell'ente sarebbe quello dell'interesse, elemento da porsi peraltro solo in relazione alla condotta e non anche all'evento del reato (così, tra le altre, la sentenza del GUP di Cagliari n. 1188/11 del 4 luglio 2011, giudice Altieri), essendo pacifico che alcuna azienda potrebbe mai avere interesse - né tanto meno ricavare alcun vantaggio - dal verificarsi di fatti di lesioni o dalla morte dei lavoratori, con violazione della normativa antiinfortunistica, fatti i quali, con ogni evidenza, sono fonte per l'azienda di numerosi e considerevoli danni di varia natura. 
In questi casi, anche secondo il GUP di Cagliari, l'interesse dell'ente idoneo a fondare una sua responsabilità ex decreto 231 è rappresentato da una tensione finalistica verso un risparmio d'impresa, anche indipendentemente dal fatto che tale obiettivo si sia effettivamente raggiunto o meno.

Si è peraltro poi ulteriormente precisato (si veda ad esempio la sentenza del GUP di Tolmezzo, n. 18/12, depositata il 3/2/2012) come, in quest' ambito, non assumano rilievo le violazioni che non siano frutto di esplicite deliberazioni volitive finalisticamente orientate a soddisfare l' interesse dell'ente, considerandosi pertanto irrilevanti profili di colpa quali la semplice imperizia, ovvero la mera sottovalutazione dei rischi oppure ancora la non adeguata considerazione o esecuzione delle misure preventive da assumere, ovvero la violazione, avvenuta in via episodica e in ambito locale e decentrato, di procedure operative vigenti in azienda o di sistemi di sicurezza vigenti nel contesto aziendale e idonei, in una prospettiva ex ante, a prevenire l'evento.

Sotto questo profilo non è chi non veda quanto profondamente differenti siano i criteri di ascrizione della responsabilità dell'ente rispetto ai criteri penalistici di imputazione della responsabilità in relazione a tale tipologia di reati .

\section{Responsabilità per fatti commessi da soggetti "apicali" e da "sottoposti".}

Ciò premesso, il decreto stabilisce che, qualora il reato presupposto sia stato commesso da soggetti apicali, l'ente è esente da responsabilità qualora provi che (a) l'organo gestorio ha adottato ed efficacemente attuato, prima della commissione del fatto, modelli di organizzazione e gestione idonei a prevenire reati della specie di quello verificatosi; (b), è stato costituito un organismo di vigilanza dotato di poteri autonomi di iniziativa e controllo, cui è stato affidato il compito di vigilare sul funzionamento e l'osservanza dei modelli nonché di curarne l' aggiornamento; (c) i soggetti cd. apicali hanno commesso il reato eludendo fraudolentemente i modelli; (d) non vi è stata omessa o insufficiente vigilanza da parte dell'organismo di vigilanza.

Qualora invece il reato presupposto sia contestato ai soggetti sottoposti, l'ente risponde a titolo di responsabilità amministrativa, qualora la commissione della violazione sia stata resa possibile dall'inosservanza degli obblighi di direzione e di vigilanza; in questo caso tuttavia l'inosservanza è esclusa se l'ente, prima della commissione del reato, ha adottato ed efficacemente attuato un modello di gestione, organizzazione e controllo idoneo a prevenire reati della specie di quello verificatosi.

Nel caso quindi di reato presupposto contestato a soggetti cd. apicali sarà l'ente a dover fornire la prova relativa all'adozione ed efficace attuazione del modello, alla costituzione di un organismo di vigilanza autonomo ed efficiente, che non abbia omesso di vigilare ovvero che non abbia vigilato in maniera insufficiente, e infine che coloro che hanno commesso il reato lo hanno fatto eludendo fraudolentemente i modelli di organizzazione e gestione.

Qualora invece il reato si assuma commesso da soggetti cd. sottoposti, sarà onere della pubblica accusa dimostrare che questo è stato reso possibile dall'inosservanza degli obblighi di direzione e di vigilanza.

Ora, al dì là della prova effettivamente diabolica richiesta dalla lett. c ) dell'art. 6 comma 1 del decreto (ovvero quella relativa alla fraudolenta elusione dei modelli da parte dei soggetti cd. apicali), prova che ben difficilmente si ritiene poter essere fornita nel termini stringenti definiti dalla norma, ci si è chiesti se la 
disciplina dettata per l' ipotesi di reato presupposto commesso da soggetti apicali comporti, per la difesa dell'ente, un'inversione dell' onere della prova.

Al fine di preservare la compatibilità del sistema introdotto dal decreto 231 con i principi posti a fondamento della responsabilità in sede penale e soprattutto delle modalità di accertamento della medesima nel processo e, in definitiva, della previsione costituzionale della presunzione di non colpevolezza, si è detto che in tali casi non vi sarebbe un'effettiva inversione dell'onere della prova, ma un interesse dell'ente a provare ciò che nei fatti ha efficacia esimente.

Tale impostazione pare toccare effettivamente il cuore e il fondamento della responsabilità dell'ente: con il decreto 231 l'ente è sostanzialmente chiamato a collaborare con l'autorità statuale affinché si possa pervenire al risultato di ridurre al minimo il rischio della commissione di reati .

\section{Una possibile conclusione sulle ragioni della rimproverabilità dell'ente.}

L'ente è quindi chiamato a cooperare con la pubblica autorità nell impegno volto alla prevenzione dei reati ed è chiamato a far questo organizzandosi adeguatamente, adottando così dei modelli che, tenendo conto della specifica realtà imprenditoriale in cui esso opera nonché delle fattispecie di reato espressamente considerate nel decreto, identifichino e definiscano procedure e modalità operative interne idonee a minimizzare - essendo chiaro che non sarebbe obiettivo realistico la neutralizzazione - il rischio della commissione dei reati.

In questo modo l'ente contribuisce altresì a riempire di contenuto e di significato il precetto in qualche modo lasciato - deliberatamente - in bianco dal legislatore: quest' ultimo, infatti, non impone all'impresa l'adozione del modello né tanto meno stabilisce, se non con indicazioni di carattere assolutamente generale quali quelle contenute negli artt. 6 e 7 del medesimo, come questo debba essere redatto e quali specifiche regole debba contenere, lasciando questo compito ad ogni impresa, nella specificità della sua attività, della sua organizzazione interna e della sua governance.

Si potrebbe anche osservare come l'adozione del modello possa costituire un'occasione, per l' impresa, per procedere ad una razionalizzazione e migliore organizzazione delle procedure interne: l'elaborazione del modello, infatti, presuppone necessariamente, in prima battuta, una completa identificazione di tutti i processi e le funzioni aziendali, nonché delle regole e procedure già applicate in azienda, quindi una mappatura dei rischi presenti in azienda alla luce dei reati presupposto presi in considerazione dal decreto, ed infine l'elaborazione di specifici protocolli volti a disciplinare le modalità di assunzione e di esecuzione delle determinazioni dell'ente al fine della prevenzione dei reati.

Pertanto, nell' ambito di una normativa volta a stimolare l'agire dell'ente improntato a principi di legalità se non addirittura di eticità delle proprie scelte, l'attività finalizzata all'adozione del modello, alla sua attuazione efficace e al continuo adeguamento dello stesso ai mutamenti aziendali nonché alle modifiche normative, ed infine l'attività di monitoraggio svolta dall'organismo di vigilanza potrebbe avere, se attuata correttamente e secondo criteri di adeguatezza alla concreta realtà imprenditoriale, effetti positivi in termini di migliore organizzazione, migliore gestione delle risorse e costante verifica della correttezza delle funzioni e dei processi. 
Ecco quindi come, a modesto avviso di chi scrive, proprio qui possa essere rinvenuto il fondamento e il nucleo della responsabilità “amministrativa “ dell'ente, che appare allora non più come una responsabilità per fatto altrui o come una responsabilità oggettiva, ma come una responsabilità per fatto proprio, laddove il fatto addebitabile all'ente consiste appunto nel non essersi saputo adeguatamente organizzare allo scopo di ridurre al minimo il rischio di commissione di reati.

La sentenza di Cassazione, sez. VI penale, n. 36083 del 9/7/2009, depositata il 17/9/2009, afferma appunto che il decreto 231 ha introdotto una forma normativa di colpevolezza, che è per fatto proprio e non altrui - rispettosa quindi dell' art. 27 Cost. - e per la quale la rimproverabilità risiede appunto nel non essersi saputo l'ente adeguatamente organizzare e disciplinare affinché venisse evitata la commissione dei reati previsti dal decreto, qualora questi, in virtù del rapporto di immedesimazione organica, siano stati commessi nell'interesse ovvero a vantaggio dell'ente medesimo.

Devesi peraltro osservare che - anche in contrasto su quanto sul punto affermato dalla sentenza da ultimo citata - la responsabilità ex d. lgs. 231 non è tuttavia responsabilità per la sola mancata adozione del modello (che infatti la legge non impone all'ente, limitandosi a prevedere che la sua esistenza ed efficace attuazione, alle condizioni previste dalla legge, possa avere efficacia esimente); anche in mancanza del modello, a fini difensivi dell'ente, debbono comunque sempre soccorrere i criteri dellinteresse o vantaggio, oltre a considerare che, naturalmente, le pronunce assolutorie nel merito nei confronti dell'imputato persona fisica per il reato presupposto (non però la circostanza per cui l'autore del reato non sia stato identificato o non sia imputabile, né le pronunce di estinzione del reato per cause diverse dall'amnistia, come espressamente previsto dall' art. 8 del decreto - il che ulteriormente depone nel senso del carattere autonomo della responsabilità dell'ente rispetto a quella dell' autore del reato presupposto) debbono necessariamente condurre ad analoga pronuncia nei confronti dell'ente.

\section{Riferimenti bibliografici.}

- Canzio G., Serqua L.D.,m Luparia L. (a cura di), Diritto penale della società. Profili sostanżali e processuali, 2 tomi, Cedam, Padova, 2014.

- Corso S.M., Codice della responsabilità "da reato" degli enti - annotato con la giurisprudenza, II ediz., Giappichelli, Torino, 2014. 